\title{
A Facile $\mathrm{Cu}(\mathrm{I})$-Catalyzed Oxidative Coupling of Anilines to Azo Compounds and Hydrazines with Diaziridinone under Mild Conditions
}

\author{
Yingguang Zhu and Yian Shi \\ Department of Chemistry, Colorado State University, Fort Collins, Colorado 80523, United States
}

\section{Abstract}

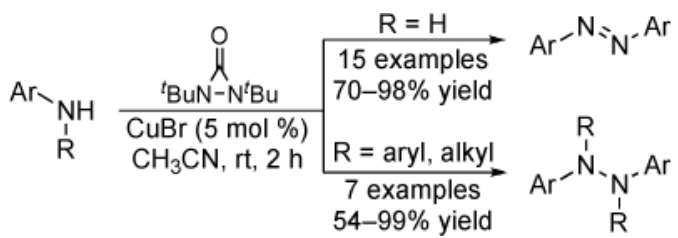

A mild and highly efficient $\mathrm{Cu}(\mathrm{I})$-catalyzed oxidative coupling of anilines is described. Various primary and secondary anilines can be efficiently coupled under mild conditions to the corresponding azo compounds and hydrazines in high yields. This method provides a direct and practical access to these compounds, and is also amenable to gram scale with no special precautions to exclude air or moisture.

Development of efficient methods for the synthesis of azo compounds is highly important as this class of molecules can be used as organic dyes, ${ }^{1}$ indicators, ${ }^{2}$ radical reaction initiators, ${ }^{3}$ nonlinear optics, ${ }^{4}$ chemosensors, ${ }^{5}$ and photochemical switches. ${ }^{6}$ Various synthetic methods ${ }^{7}$ have been developed including oxidation of hydrazines, ${ }^{8}$ reduction of azoxybenzenes, ${ }^{9}$ coupling of aryl diazonium salts with nucleophiles, ${ }^{10}$ coupling of primary anilines with aromatic nitroso compounds, ${ }^{11}$ and reductive coupling of nitroaromatics. ${ }^{12}$ Azo compounds can also be obtained by oxidative coupling of anilines using stoichiometric oxidants, such as mercury(II) oxide, ${ }^{13}$ manganese salts, ${ }^{14}$ lead tetraacetate, ${ }^{15}$ ferrate salts, ${ }^{16}$ and tert-butyl hypoiodite. ${ }^{17}$ Metal-catalyzed oxidative coupling of anilines using $\mathrm{O}_{2}$ has also been reported. ${ }^{18}$

In our earlier studies, di-tert-butyldiaziridinone (1) ${ }^{19,20}$ has been shown to be a highly effective nitrogen source for the diamination of olefins using $\mathrm{Pd}(0)^{21}$ or $\mathrm{Cu}(\mathrm{I})^{22}$ as catalysts. Very recently, we have shown that diaziridinone 1 can oxidize alcohols to aldehydes and ketones using $\mathrm{CuBr}$ as catalyst. ${ }^{23}$ In our efforts to further explore the synthetic utility of diaziridinone, we have found that anilines can be efficiently oxidized to azo compounds and hydrazines using $\mathrm{CuBr}$ catalyst and diaziridinone $\mathbf{1}$. Herein we wish to report our preliminary results on this subject.

When phenylamine (2a) was treated with di-tert-butyldiaziridinone (1) and $5 \mathrm{~mol} \% \mathrm{CuBr}$ in $\mathrm{CH}_{3} \mathrm{CN}$ at $\mathrm{rt}$ under air for $2 \mathrm{~h}$, azobenzene $3 \mathrm{a}$ was cleanly formed and isolated in $93 \%$ yield (Scheme 1). Similar yield (94\%) was obtained when this reaction was carried out under

yian@lamar.colostate.edu.

Supporting Information Available. Experimental procedures, characterization data, and NMR spectra. This material is available free of charge via the Internet at http://pubs.acs.org. 
argon. Both di-tert-butyldiaziridinone (1) and $\mathrm{Cu}(\mathrm{I})$ catalyst are required for the transformation as no oxidation was observed in the absence of $\mathbf{1}$ or $\mathrm{CuBr}$ under air for $24 \mathrm{~h}$ (Scheme 1).

As shown in Scheme 2, this oxidative coupling can be extended to a variety of primary anilines bearing either electron-donating or withdrawing groups at the para, meta, and ortho positions on the phenyl ring, giving the corresponding azo compounds in 70-98\% yield (Scheme 2, 3a-3n). Halo-substituted azobenzenes can be obtained in high yields from the corresponding anilines, and no Ullmann-type $\mathrm{C}-\mathrm{N}$ coupling byproducts were detected (Scheme 2, 3j-3l). As illustrated in the case of 4-bromoaniline (2l), azo product $\mathbf{3 l}$ can be obtained on gram scale in $91 \%$ yield (Scheme 2). As shown in Scheme 3, the oxidative coupling of aniline proceeded faster than the oxidation of an alcohol. Azobenzene $\mathbf{3 o}$ was obtained when 20 was treated with 1.1 equiv of diaziridinone 1 and $10 \mathrm{~mol} \% \mathrm{CuBr}$ in $\mathrm{CH}_{3} \mathrm{CN}$ at $\mathrm{rt}$ for $2 \mathrm{~h}$. Both amine and alcohol were oxidized with 2.1 equiv of $\mathbf{1}$, giving diketone 3o' in $91 \%$ yield.

When diphenylamine (4a) was subjected to the reaction conditions, tetraphenylhydrazine (5a) was obtained in $60 \%$ yield (Scheme 4 ). This coupling process is also applicable to various other secondary anilines, giving the corresponding tetrasubstituted hydrazines in 5499\% yield (Scheme 4, 5b-5g). As illustrated in the case of bis(4-bromophenyl)amine (4f), hydrazine $\mathbf{5 f}$ can be obtained on gram scale in $99 \%$ yield (Scheme 4). Hydrazines are a class of very important compounds. ${ }^{24}$ Substituted hydrazines are usually prepared by derivatization of simple hydrazine precursors. ${ }^{24,25}$ There are few methods reported in the literature for the direct synthesis of substituted hydrazines from readily available secondary anilines. ${ }^{26}$ The current method provides a viable process for the synthesis of tetrasubstituted hydrazines.

In order to better understand the mode of reactivity, 1,2-diphenylhydrazine (6a) was subjected to the reaction conditions. Azobenzene 3a was obtained in $96 \%$ yield in only 5 min (Scheme 5), suggesting that 6a is a possible intermediate for the oxidative coupling of aniline. Attempts to isolate intermediate $\mathbf{6 a}$ in the coupling of aniline $\mathbf{2 a}$ using 0.55 equiv of 1 proved unsuccessful, yielding azobenzene $3 \mathbf{a}$ in $47 \%$ yield along with unreacted starting material (Scheme 5).

While a precise understanding of the reaction mechanism awaits further study, a plausible catalytic pathway for the oxidative coupling of anilines is proposed in Scheme 6. The N-N bond of di-tert-butyldiaziridinone (1) is reductively cleaved by $\mathrm{CuBr}$ to form $\mathrm{Cu}$ (II) nitrogen radical $\mathbf{A}^{27,28}$ and/or $\mathrm{Cu}(\mathrm{III})$ species $\mathbf{B}^{22 \mathrm{e}, \mathrm{f}}$, which reacts with aniline $\mathbf{2}$ to generate the corresponding nitrogen radical $\mathbf{C}^{28}$ and/or $\mathbf{C u}(\mathrm{III})$ species $\mathbf{D}$. Hydrazine $\mathbf{6}$ is subsequently formed via the dimerization of radical $\mathbf{C}$ or reductive elimination of species $\mathbf{D}$. The dehydrogenation of $\mathbf{6}$ by $\mathrm{Cu}$ species $\mathbf{A}$ and/or $\mathbf{B}$ forms product $\mathbf{3}$.

In summary, we have developed a novel oxidative coupling of anilines using $\mathrm{CuBr}$ as catalyst and di-tert-butyldiaziridinone (1) as oxidant. Various primary and secondary anilines can be efficiently coupled under mild conditions to give the corresponding azo compounds and hydrazines in high yields. This reaction is operationally simple and is amenable to gram scale. The results further illustrate the synthetic potential of diaziridinone as a versatile oxidation reagent. Development of other reaction processes with this class of compounds is currently underway.

\section{Supplementary Material}

Refer to Web version on PubMed Central for supplementary material. 


\section{Acknowledgments}

We are grateful for the generous financial support from the General Medical Sciences of the National Institutes of Health (GM083944-05).

\section{References}

1. For leading reviews, see:(a) Hunger, K. Industrial Dyes: Chemistry, Properties, Applications. Wiley-VCH; Weinheim: 2003. (b) Bafana A, Devi SS, Chakrabarti T. Environ Rev. 2011; 19:350.

2. For leading reviews, see:(a) Anderson RG, Nickless G. Analyst. 1967; 92:207. [PubMed: 4859975] (b) Ashutosh NDP, Mehrotra JK. Colourage. 1979; 26:25.

3. For leading reviews, see:(a) Sheppard CS. Encycl Polym Sci Eng. 1985; 2:143.(b) Bernaerts KV, Du Prez FE. Prog Polym Sci. 2006; 31:671.

4. For leading references, see:(a) Burland DM, Miller RD, Walsh CA. Chem Rev. 1994; 94:31.(b) Kanis DR, Ratner MA, Marks TJ. Chem Rev. 1994; 94:195.(c) Ishow E, Bellaïche C, Bouteiller L, Nakatani K, Delaire JA. J Am Chem Soc. 2003; 125:15744. [PubMed: 14677955] (d) Yesodha SK, Pillai CKS, Tsutsumi N. Prog Polym Sci. 2004; 29:45.(e) Qiu F, Cao Y, Xu H, Jiang Y, Zhou Y, Liu J. Dyes Pigm. 2007; 75:454.(f) Sahraoui B, Luc J, Meghea A, Czaplicki R, Fillaut JL, Migalska-Zalas A. J Opt A: Pure Appl Opt. 2009; 11:24005.(g) Papagiannouli I, Iliopoulos K, Gindre D, Sahraoui B, Krupka O, Smokal V, Kolendo A, Couris S. Chem Phys Lett. 2012; 554:107. (h) Ashraf M, Teshome A, Kay AJ, Gainsford GJ, Bhuiyan MDH, Asselberghs I, Clays K. Dyes Pigm. 2012; 95:455.

5. For leading references, see:(a) DiCesare N, Lakowicz JR. Org Lett. 2001; 3:3891. [PubMed: 11720562] (b) Bhardwaj VK, Singh N, Hundal MS, Hundal G. Tetrahedron. 2006; 62:7878.(c) Isaad J, Perwuelz A. Tetrahedron Lett. 2010; 51:5810.(d) Chang KC, Su IH, Wang YY, Chung WS. Eur J Org Chem. 2010:4700.

6. For leading references, see:(a) Kumar GS, Neckers DC. Chem Rev. 1989; 89:1915.(b) Harvey AJ, Abell AD. Tetrahedron. 2000; 56:9763.(c) Dürr, H.; Bouas-Laurent, H. Photochromism: Molecules and Systems. Elsevier; Amsterdam: 2003. (d) Ghosh S, Banthia AK, Chen Z. Tetrahedron. 2005; 61:2889.

7. For leading reviews on the synthesis of azo compounds, see:(a) Hamon F, Djedaini-Pilard F, Barbot F, Len C. Tetrahedron. 2009; 65:10105.(b) Merino E. Chem Soc Rev. 2011; 40:3835. [PubMed: 21409258]

8. For leading references, see:(a) Whalley BJP, Evans HGV, Winkler CA. Can J Chem. 1956; 34:1154. (b) Blackadder DA, Hinshelwood C. J Chem Soc. 1957:2904.(c) Dimroth K, Tüncher W. Synthesis. 1977:339.(d) Hyatt JA. Tetrahedron Lett. 1977; 18:141.(e) Barton DHR, Lester DJ, Ley SV. J Chem Soc Chem Commun. 1978:276.(f) Back TG. J Chem Soc Chem Commun. 1978:278.(g) Hoffman RV, Kumar A. J Org Chem. 1984; 49:4014.(h) Billera CF, Dunn TB, Barry DA, Engel PS. J Org Chem. 1998; 63:9763.(i) Shi L, Pan F, Jia X, Wang Y. Synth Commun. 2001; 31:1691.(j) Li XC, Wang YL, Wang JY. J Chem Res. 2002:540.(k) Lim YK, Lee KS, Cho CG. Org Lett. 2003; 5:979. [PubMed: 12659553] (1) Barth M, Shah STA, Rademann J. Tetrahedron. 2004; 60:8703.(m) Kim SSB, Hommer RB, Cannon RD. Bull Korean Chem Soc. 2006; 27:255.(n) Drug E, Gozin M. J Am Chem Soc. 2007; 129:13784. [PubMed: 17958362] (o) Mihara M, Nakai T, Iwai T, Ito T, Mizuno T. Synlett. 2007:2124.(p) Gao W, He Z, Qian Y, Zhao J, Huang Y. Chem Sci. 2012; 3:883.

9. For leading references, see:(a) Mukaiyama T, Nambu H, Okamoto M. J Org Chem. 1962; 27:3651. (b) Olah GA, BalaramGupta BG, Narang SC. J Org Chem. 1978; 43:4503.(c) Sanz R, Escribano J, Fernández Y, Aguado R, Pedrosa MR, Arnáiz FJ. Synlett. 2005:1389.(d) Saini A, Kumar S, Sandhu JS. Synlett. 2006:395.

10. For leading references, see:(a) Barbero M, Degani I, Dughera S, Fochi R, Perracino P. Synthesis. 1998:1235.(b) Merrington J, James M, Bradley M. Chem Commun. 2002:140.(c) Tomasulo M, Raymo FM. Org Lett. 2005; 7:4633. [PubMed: 16209497] (d) Tsai WJ, Shiao YJ, Lin SJ, Chiou WF, Lin LC, Yang TH, Teng CM, Wu TS, Yang LM. Bioorg Med Chem Lett. 2006; 16:4440. [PubMed: 16814546] (e) Barbero M, Cadamuro S, Dughera S, Giaveno C. Eur J Org Chem. 2006:4884.(f) Lee MH, Cho BK, Yoon J, Kim JS. Org Lett. 2007; 9:4515. [PubMed: 17910464] (g) Dabbagh HA, Teimouri A, Chermahini AN. Dyes Pigm. 2007; 73:239.(h) He Y, He W, Wei R, Chen Z, Wang X. Chem Commun. 2012:1036. 
11. For leading references, see:(a) Krageloh K, Anderson GH, Stang PJ. J Am Chem Soc. 1984; 106:6015.(b) Davey MH, Lee VY, Miller RD, Marks TJ. J Org Chem. 1999; 64:4976. [PubMed: 11674586] (c) Park SB, Standaert RF. Tetrahedron Lett. 1999; 40:6557.(d) Badjić JD, Kostić NM. J Mater Chem. 2001; 11:408.(e) Nihei M, Kurihara M, Mizutani J, Nishihara H. J Am Chem Soc. 2003; 125:2964. [PubMed: 12617664] (f) Wang S, Wang X, Li L, Advincula RC. J Org Chem. 2004; 69:9073. [PubMed: 15609940] (g) Priewisch B, Rück-Braun K. J Org Chem. 2005; 70:2350. [PubMed: 15760229] (h) Tie C, Gallucci JC, Parquette JR. J Am Chem Soc. 2006; 128:1162. [PubMed: 16433532] (i) Dong SL, Löweneck M, Schrader TE, Schreier WJ, Zinth W, Moroder L, Renner C. Chem-Eur J. 2006; 12:1114. [PubMed: 16294349] (j) Standaert RF, Park SB. J Org Chem. 2006; 71:7952. [PubMed: 17025282] (k) Shee B, Pratihar JL, Chattopadhyay S. Polyhedron. 2006; 25:2513.(l) Peters MV, Stoll RS, Goddard R, Buth G, Hecht S. J Org Chem. 2006; 71:7840. [PubMed: 16995694] (m) Harvey JH, Butler BK, Trauner D. Tetrahedron Lett. 2007; 48:1661.(n) Liu Z, Jiang M. J Mater Chem. 2007; 17:4249.(o) Kamei T, Kudo M, Akiyama H, Wada M, Nagasawa J, Funahashi M, Tamaoki N, Uyeda TQP. Eur J Org Chem. 2007:1846.(p) Zarwell S, Rück-Braun K. Tetrahedron Lett. 2008; 49:4020.(q) Lee GW, Kim NK, Jeong KS. Org Lett. 2010; 12:2634. [PubMed: 20446657] (r) Bellotto S, Reuter R, Heinis C, Wegner HA. J Org Chem. 2011; 76:9826. [PubMed: 22004310]

12. For leading references, see:(a) Chung TF, Wu YM, Cheng CH. J Org Chem. 1984; 49:1215.(b) Ohe K, Uemura S, Sugita N, Masuda H, Taga T. J Org Chem. 1989; 54:4169.(c) Wada S, Urano M, Suzuki H. J Org Chem. 2002; 67:8254. [PubMed: 12423164] (d) Srinivasa GR, Abiraj K, Gowda DC. Tetrahedron Lett. 2003; 44:5835.(e) Sakai N, Fujii K, Nabeshima S, Ikeda R, Konakahara T. Chem Commun. 2010:3173.(f) Zhu H, Ke X, Yang X, Sarina S, Liu H. Angew Chem Int Ed. 2010; 49:9657.(g) Hu L, Cao X, Shi L, Qi F, Guo Z, Lu J, Gu H. Org Lett. 2011; 13:5640. [PubMed: 21939197]

13. For leading references, see:(a) Orito K, Hatakeyama T, Takeo M, Uchiito S, Tokuda M, Suginome H. Tetrahedron. 1998; 54:8403.(b) Flatt AK, Dirk SM, Henderson JC, Shen DE, Su J, Reed MA, Tour JM. Tetrahedron. 2003; 59:8555.(c) Farhadi S, Zaringhadam P, Sahamieh RZ. Acta Chim Slov. 2007; 54:647.

14. For leading references, see:(a) Barakat MZ, Abdel-Wahab MF, El-Sadr MM. J Chem Soc. 1956:4685.(b) Wheeler OH, Gonzalez D. Tetrahedron. 1964; 20:189.(c) Bhatnagar I, George MV. J Org Chem. 1968; 33:2407.(d) Firouzabadi H, Vessal B, Naderi M. Tetrahedron Lett. 1982; 23:1847.(e) Firouzabadi H, Mostafavipoor Z. Bull Chem Soc Jpn. 1983; 56:914.(f) Shaabani A, Lee DG. Tetrahedron Lett. 2001; 42:5833.(g) Gilbert AM, Failli A, Shumsky J, Yang Y, Severin A, Singh G, Hu W, Keeney D, Petersen PJ, Katz AH. J Med Chem. 2006; 49:6027. [PubMed: 17004716] (h) Thorwirth R, Bernhardt F, Stolle A, Ondruschka B, Asghari J. Chem-Eur J. 2010; 16:13236. [PubMed: 20922723]

15. For leading references, see:(a) Pausacker KH, Scroggie JG. J Chem Soc. 1954:4003.(b) Baer E, Tosoni AL. J Am Chem Soc. 1956; 78:2857.

16. For leading references, see:(a) Firouzabadi H, Mohajer D, Entezari-Moghadam M. Bull Chem Soc Jpn. 1988; 61:2185.(b) Huang H, Sommerfeld D, Dunn BC, Lloyd CR, Eyring EM. J Chem Soc Dalton Trans. 2001:1301.

17. For a leading reference, see:Takeda Y, Okumura S, Minakata S. Angew Chem Int Ed. 2012; 51:7804.

18. For leading references, see:(a) Grirrane A, Corma A, García H. Science. 2008; 322:1661. [PubMed: 19074342] (b) Lu W, Xi C. Tetrahedron Lett. 2008; 49:4011.(c) Zhang C, Jiao N. Angew Chem Int Ed. 2010; 49:6174.(d) Nguyen HT, Coulembier O, Gheysen K, Martins JC, Dubois P. Macromolecules. 2012; 45:9547.(e) Hu Z, Kerton FM. Org Biomol Chem. 2012; 10:1618. [PubMed: 22231227]

19. For a leading review on diaziridinones, see:Heine, HW. The Chemistry of Heterocyclic Compounds. Hassner, A., editor. John Wiley \& Sons, Inc; New York: 1983. p. 547

20. For the preparation of di-tert-butyldiaziridinone (1), see:Du H, Zhao B, Shi Y. Org Synth. 2009; 86:315.

21. For Pd(0)-catalyzed diamination of olefins using 1, see:(a) Du H, Zhao B, Shi Y. J Am Chem Soc. 2007; 129:762. [PubMed: 17243803] (b) Du H, Yuan W, Zhao B, Shi Y. J Am Chem Soc. 2007; 129:11688. [PubMed: 17803307] (c) Du H, Yuan W, Zhao B, Shi Y. J Am Chem Soc. 2007; 129:7496. [PubMed: 17521192] (d) Du H, Zhao B, Shi Y. J Am Chem Soc. 2008; 130:8590. 
[PubMed: 18549207] (e) Zhao B, Du H, Cui S, Shi Y. J Am Chem Soc. 2010; 132:3523. [PubMed: 20166669]

22. For $\mathrm{Cu}(\mathrm{I})$-catalyzed diamination of olefins using 1, see:(a) Yuan W, Du H, Zhao B, Shi Y. Org Lett. 2007; 9:2589. [PubMed: 17536815] (b) Du H, Zhao B, Yuan W, Shi Y. Org Lett. 2008; 10:4231. [PubMed: 18763785] (c) Wen Y, Zhao B, Shi Y. Org Lett. 2009; 11:2365. [PubMed: 19408926] (d) Zhao B, Du H, Shi Y. J Org Chem. 2009; 74:8392. [PubMed: 19827794] (e) Zhao B, Peng X, Cui S, Shi Y. J Am Chem Soc. 2010; 132:11009. [PubMed: 20698659] (f) Zhao B, Peng X, Zhu Y, Ramirez TA, Cornwall RG, Shi Y. J Am Chem Soc. 2011; 133:20890. [PubMed: 22081888]

23. Zhu Y, Zhao B, Shi Y. Org Lett. 2013; 15:992. [PubMed: 23413952]

24. For leading reviews, see:(a) Schiessl, HW. Hydrazine and Its Derivatives. In: Kroschwitz, JI.; Howe-Grant, M., editors. Kirk-Othmer Encyclopedia of Chemical Technology. 4. Vol. 13. Wiley; New York: 1995. (b) Ragnarsson U. Chem Soc Rev. 2001; 30:205.

25. For leading references on the synthesis of substituted hydrazines from simple hydrazine precursors, see:(a) Mäeorg U, Grehn L, Ragnarsson U. Angew Chem Int Ed. 1996; 35:2626.(b) Alberti A, Canè F, Dembech P, Lazzari D, Ricci A, Seconi G. J Org Chem. 1996; 61:1677. [PubMed: 11667036] (c) Grehn L, Lönn H, Ragnarsson U. Chem Commun. 1997:1381.(d) Mäeorg U, Ragnarsson U. Tetrahedron Lett. 1998; 39:681.(e) Brosse N, Pinto MF, Jamart-Grégoire B. J Org Chem. 2000; 65:4370. [PubMed: 10891140] (f) Tšubrik O, Mäeorg U. Org Lett. 2001; 3:2297. [PubMed: 11463300] (g) Arterburn JB, Rao KV, Ramdas R, Dible BR. Org Lett. 2001; 3:1351. [PubMed: 11348232] (h) Brosse N, Pinto MF, Bodiguel J, Jamart-Grégoire B. J Org Chem. 2001; 66:2869. [PubMed: 11304216] (i) Tšubrik O, Mäeorg U, Sillard R, Ragnarsson U. Tetrahedron. 2004; 60:8363.(j) Bredihhin A, Groth UM, Mäeorg U. Org Lett. 2007; 9:1097. [PubMed: 17309274] (k) Bredihhin A, Mäeorg U. Org Lett. 2007; 9:4975. [PubMed: 17979277] (1) Rosamilia AE, Aricò F, Tundo P. J Org Chem. 2008; 73:1559. [PubMed: 18211082] (m) Brown MJ, Clarkson GJ, Fox DJ, Inglis GG, Shipman M. Tetrahedron Lett. 2010; 51:382.

26. For leading references, see:(a) Kajimoto T, Takahashi H, Tsuji J. Bull Chem Soc Jpn. 1982; 55:3673.(b) Joshaghani M, Rafiee E, Shahbazi F, Jafari H, Amiri S, Omidi M. ARKIVOC. 2007; i:164.(c) Yan XM, Chen ZM, Yang F, Huang ZZ. Synlett. 2011:569.(d) Zhang L, Xia J, Li Q, Li X, Wang S. Organometallics. 2011; 30:375.

27. For a leading review on metal-promoted radical reactions, see:Iqbal J, Bhatia B, Nayyar NK. Chem Rev. 1994; 94:519.

28. For leading reviews on nitrogen-centered radicals, see:(a) Danen WC, Neugebauer FA. Angew Chem Int Ed. 1975; 14:783.(b) Mackiewicz P, Furstoss R. Tetrahedron. 1978; 34:3241.(c) Stella L. Angew Chem Int Ed. 1983; 22:337.(d) Esker JL, Newcomb M. Adv Heterocycl Chem. 1993; 58:1.(e) Zard SZ. Synlett. 1996:1148.(f) Stella, L. Radicals in Organic Synthesis. Renaud, P.; Sibi, MP., editors. Vol. 2. Wiley-VCH; Weinheim: 2001. p. 407(g) Zard SZ. Chem Soc Rev. 2008; 37:1603. [PubMed: 18648685] (h) Minozzi M, Nanni D, Spagnolo P. Chem-Eur J. 2009; 15:7830. [PubMed: 19551769] 


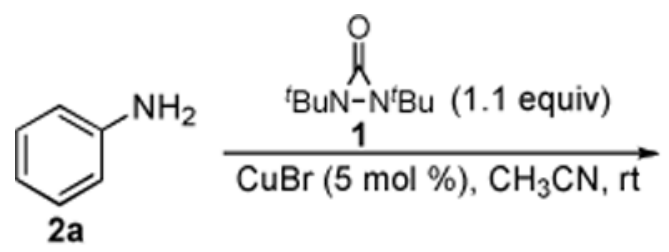

a) under air, $2 \mathrm{~h}$

b) under $\mathrm{Ar}, 2 \mathrm{~h}$

c) no diaziridinone 1, under air, $24 \mathrm{~h}$

d) no $\mathrm{CuBr}$, under air, $24 \mathrm{~h}$<smiles>c1ccc(/N=N/c2ccccc2)cc1</smiles>

$93 \%$ yield

$94 \%$ yield no reaction no reaction

Scheme 1. 


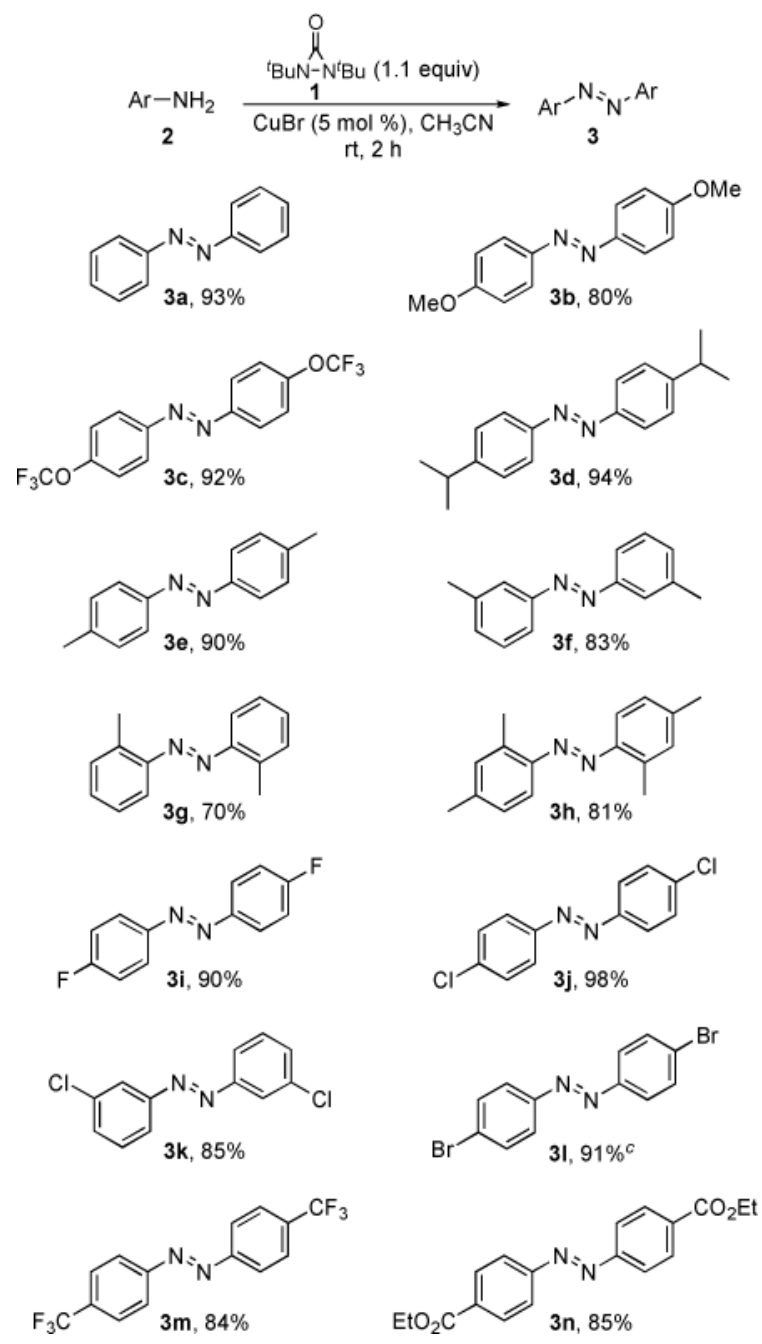

Scheme 2.

Oxidative Coupling of Primary Anilines ${ }^{a, b}$

${ }^{a}$ All reactions were carried out with primary aniline $2(0.80 \mathrm{mmol}), \mathbf{1}(0.88 \mathrm{mmol})$, and $\mathrm{CuBr}(0.040 \mathrm{mmol})$ in $\mathrm{CH}_{3} \mathrm{CN}(1.6 \mathrm{~mL})$ at $\mathrm{rt}$ for $2 \mathrm{~h}$ unless otherwise stated. ${ }^{b}$ Isolated yield based on primary aniline $2 .^{\circ}$ The reaction was carried out on $8.0 \mathrm{mmol}$ scale. 


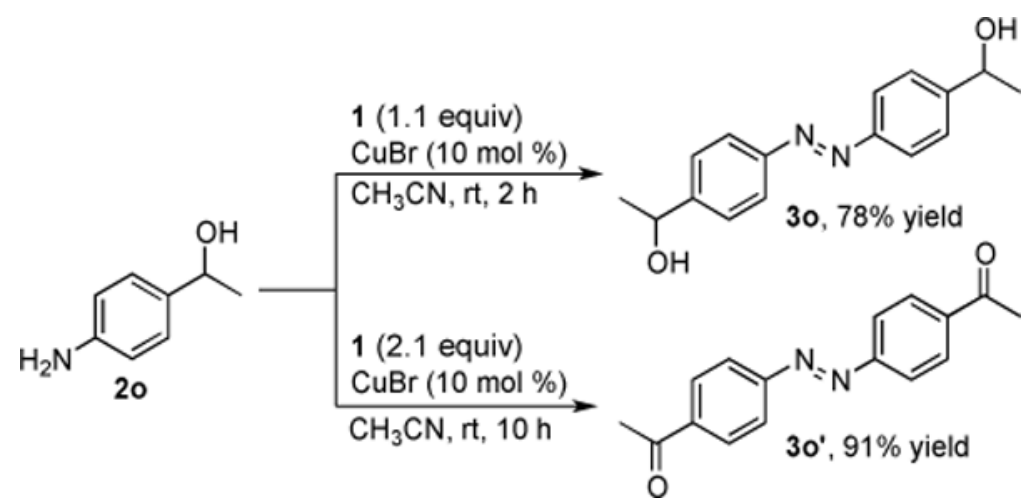

Scheme 3.

Org Lett. Author manuscript; available in PMC 2014 April 19. 


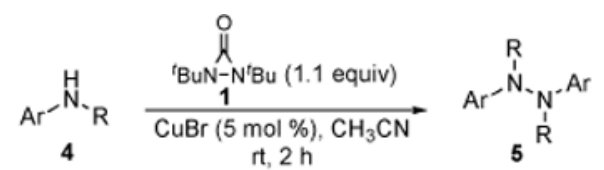<smiles>CC(C)(C)c1ccc(N(c2ccccc2)c2ccccc2)cc1</smiles><smiles>Cc1ccc(N(c2ccc(C)cc2)N(c2ccc(C)cc2)c2ccc(C)cc2)cc1</smiles><smiles>Cc1ccc(N(c2ccccc2)N(c2ccccc2)c2ccc(C)cc2)cc1</smiles><smiles>Cc1cccc(N(c2ccccc2)N(c2ccccc2)c2ccccc2)c1</smiles><smiles>Brc1ccc(N(c2ccccc2)N(c2ccccc2)c2ccc(Br)cc2)cc1</smiles><smiles>CC(C)(C)C(C)(C)C</smiles><smiles>CN(c1ccc(Br)cc1)N(C)c1ccc(Br)cc1</smiles>

Scheme 4.

Oxidative Coupling of Secondary Anilines ${ }^{a, b}$

${ }^{a}$ All reactions were carried out with secondary aniline $4(0.80 \mathrm{mmol}), \mathbf{1}(0.88 \mathrm{mmol})$, and $\mathrm{CuBr}(0.040 \mathrm{mmol})$ in $\mathrm{CH}_{3} \mathrm{CN}(1.6 \mathrm{~mL})$ at $\mathrm{rt}$ for $2 \mathrm{~h}$ unless otherwise stated. ${ }^{b}$ Isolated yield based on secondary aniline 4 . $^{\circ}$ The reaction was carried out on $8.0 \mathrm{mmol}$ scale. 


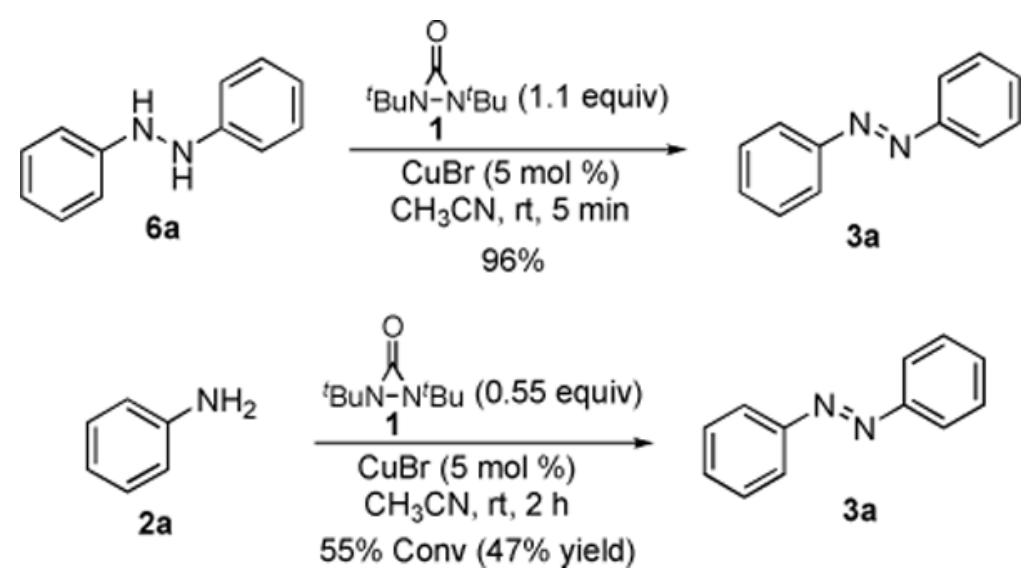

Scheme 5. 

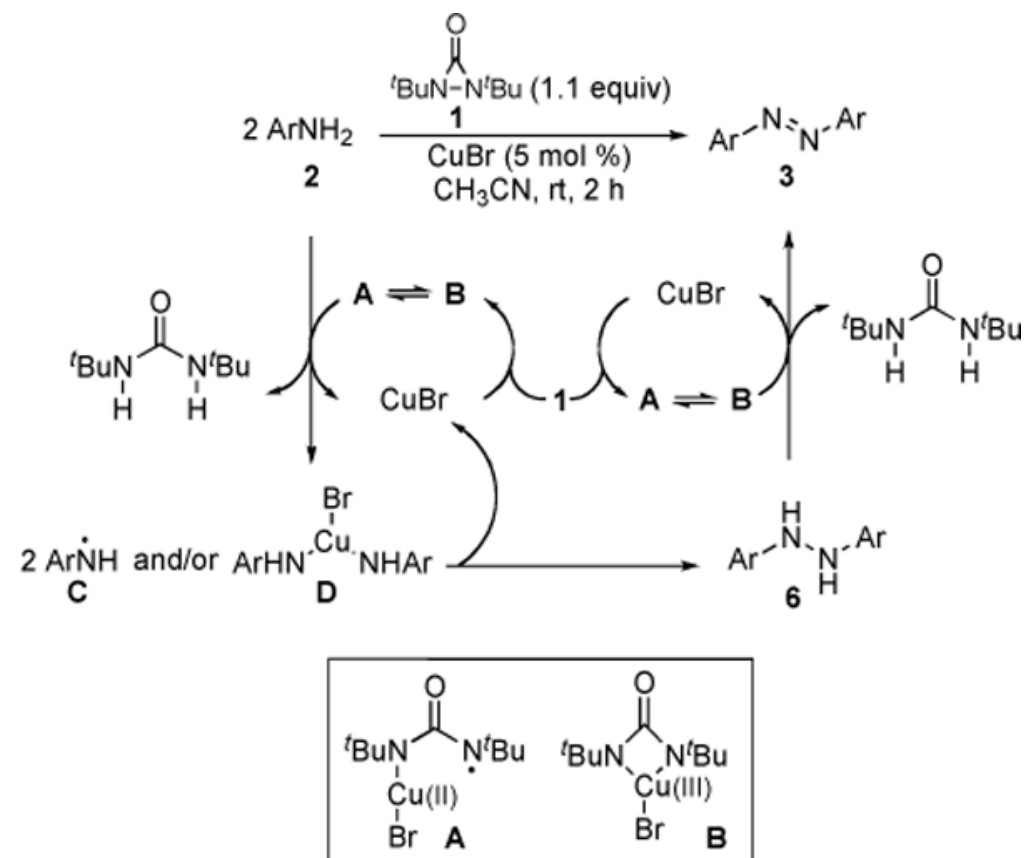

Scheme 6.

Proposed Catalytic Cycle for the Oxidative Coupling 